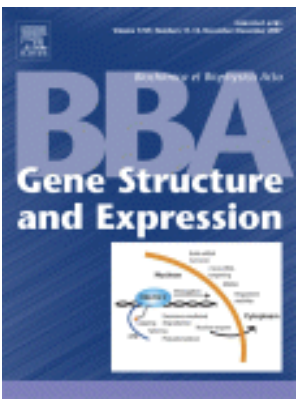

Short sequence-paper

\title{
Clusters of 5S rRNAs in the intergenic region of ubiquitin genes in Tetrahymena pyriformis
}

- Paulo Guerreiro,

- Ana Neves,

- Claudina Rodrigues-Pousada

Laboratório de Genética Molecular, Instituto Gulbenkian de Ciência, Apartado 14, 2781 Oeiras Codex Portugal

\section{Abstract}

Here, we report the molecular analysis of two independent $5 S$ rRNA clusters found in the intergenic region of two ubiquitin genomic clones isolated from Tetrahymena pyriformis. Each cluster contains two 120-bp-long coding regions organized in tandem with 142/145-bp-long spacers.

\section{Keywords}

- Genome;

- Ubiquitin;

- Intergenic region;

- Ribosomal gene, 5S;

- rRNA cluster;

- (T. pyriformis) 